High Temperature Material Processes 22(2-3):v-vi (2018)

\title{
PREFACE: ADVANCES IN COMPUTATIONAL HEAT TRANSFER (CHT-17)
}

It is a great pleasure and honor to present this special issue of High Temperature Material Processes based on a selection of papers presented at the 7th International Symposium on Advances in Computational Heat Transfer (CHT-17), which was sponsored by the International Centre for Heat and Mass Transfer (ICHMT), the School of Engineering, Rutgers University, and the Università degli Studi della Campania "Luigi Vanvitelli." The symposium took place during 28 May-1 June 2017 in Napoli, Italy. It was held at the Conference Centre of University of Napoli "Federico II," Napoli, Italy. This symposium is a continuation of the series of symposia held at various places around the world, including Cesme, Turkey; Marrakesh, Morocco; Bath, UK; and Piscataway, New Jersey, USA. It focuses on computational heat transfer and covers a wide range of topics of both fundamental and applied interest.

The main objective of the present symposium was to provide a forum for the presentation and exchange of new ideas and trends, methods, and results emerging from the use of computational techniques for solving a wide range of problems in different heat transfer areas. Researchers from academia and industry came together, along with students and CHT practitioners, to discuss new and emerging areas, as well as traditional fields. Besides the contributed papers, a large number of keynote and invited lectures were presented to focus on the current state of the art and future needs.

During the symposium, there were 5 plenary talks, 16 keynote lectures, 157 oral presentations, and 32 posters. The presentations were in 34 regular sessions with one panel on radiation. The number of participants was over 230 and about 30 countries around the world were represented. Moreover, the symposium included a Memorial to Professor D. B. Spalding, who had passed away earlier in the year, with one technical talk and 3 sessions with 10 presentations.

The authors of the contributed papers were encouraged to submit their updated and completed papers for publication in a Special Issue of High Temperature Material Processes. Several papers presented at the symposium were abstracts or extended abstracts. These were expanded to include additional details and results to obtain a full paper and the other papers were updated, modified, and expanded. The submitted papers were reviewed, following the journal procedures, and accepted on the basis of the peer reviews. The papers in this Special Issue represent a small subset of the wide diversity of papers included in the symposium. Still, a fairly wide range of topics is seen in the papers included here. They range from natural convection, phase change, radiation, and combustion to channel flow and various applications.

We would like to acknowledge the strong support provided by Professor Faruk Arinc, Secretary General of ICHMT, and other members of the staff at the Centre. We are grateful to the local Organizing Committee at Università degli Studi della Campania "Luigi Vanvitelli" and Università degli Studi di Napoli "Federico II," the Scientific Organizing Committee, and the International Scientific Committee for their advice, help, and support. Financial support from Begell House Inc., and Comsol, participant travel support from NSF, and support from the local Italian universities are gratefully acknowledged. 
Certainly, the most important contribution to the symposium is made by the authors and participants. We are very grateful to the many keynote and invited speakers for taking the time to attend the symposium and present overviews of their areas of expertise. We would also like to thank the reviewers of the papers in this Special Issue for their valuable comments and suggestions. We acknowledge the help and support provided by the Editors, Gioia Falcone and Arun K. Nayak, and the publishing staff of Begell House.

Guest Editors:

Oronzio Manca

Dipartimento di Ingegneria Industriale, Università

degli Studi della Campania "Luigi Vanvitelli" Aversa, Italy

Yogesh Jaluria

Department of Mechanical Engineering, Rutgers University

Piscataway, New Jersey, USA 